\title{
Quantum teleportation with imperfect quantum dots
}

\author{
F. Basso Basset $\mathbb{D}^{1 凶}$, F. Salusti ${ }^{1}$, L. Schweickert $\mathbb{D}^{2}$, M. B. Rota $\mathbb{D}^{1}{ }^{1}$, D. Tedeschi ${ }^{1}$, S. F. Covre da Silva ${ }^{3}$, E. Roccia ${ }^{1}$, V. Zwiller $\mathbb{D}^{2}$, \\ K. D. Jöns (D) $^{2,4}$, A. Rastelli iD $^{3}$ and R. Trotta ${ }^{1 \times}$
}

Efficient all-photonic quantum teleportation requires fast and deterministic sources of highly indistinguishable and entangled photons. Solid-state-based quantum emitters-notably semiconductor quantum dots-are a promising candidate for the role. However, despite the remarkable progress in nanofabrication, proof-of-concept demonstrations of quantum teleportation have highlighted that imperfections of the emitter still place a major roadblock in the way of applications. Here, rather than focusing on source optimization strategies, we deal with imperfections and study different teleportation protocols with the goal of identifying the one with maximal teleportation fidelity. Using a quantum dot with sub-par values of entanglement and photon indistinguishability, we show that the average teleportation fidelity can be raised from below the classical limit to $0.842(14)$, adopting a polarization-selective Bell state measurement and moderate spectral filtering. Our results, which are backed by a theoretical model that quantitatively explains the experimental findings, loosen the very stringent requirements set on the ideal entangled-photon source and highlight that imperfect quantum dots can still have a say in teleportation-based quantum communication architectures.

npj Quantum Information (2021)7:7; https://doi.org/10.1038/s41534-020-00356-0

\section{INTRODUCTION}

Initially pursued as one of the most surprising consequences of the concept of entanglement in quantum information science ${ }^{1,2}$, quantum teleportation has been increasingly investigated as a basic element for various applications in quantum technology ${ }^{3}$. Its photonic implementation is essential to the exchange of qubits over long distances and in fully-fledged quantum networks ${ }^{4,5}$.

Which technical platform has the better application potential is a question open for debate, even in the light of the impressive achievements reached with state-of-the-art solutions ${ }^{6}$. A main requirement concerns the generation of entangled photons ${ }^{7}$. In order to reduce losses and enable complex functionalities, a deterministic photon source would be a fundamental asset, which is a major obstacle for probabilistic sources ${ }^{8}$.

Semiconductor quantum dots (QDs) are a promising candidate for achieving on-demand operation ${ }^{9}$. In recent years, entangled QD photons have been used in seminal demonstrations of quantum teleportation to transfer qubits encoded in either an attenuated laser pulse $\mathrm{s}^{10-12}$ or a single photon generated on demand by the same $\mathrm{QD}^{13}$. While these results paved the way for the following generation of four-photon experiments ${ }^{14,15}$, imperfections of the source still played a significant role in forcing to either work at low values of protocol fidelity or with narrow temporal post-selection.

These challenges keep motivating research on the QD fabrication, but the near-future roadmap for QD-based quantum communication points at remote QDs and poses additional demands on photon indistinguishability ${ }^{16,17}$. A better understanding of the role of specific QD imperfections in the performance of quantum communication protocols will be mandatory on the path towards real-life applications.

In this work, we study in detail the impact that imperfections in the entangled photon source have on the success of the quantum teleportation operation. We investigate different schemes, comparing the simplest that selects only one out of the four Bell states to the one that implements a polarization-selective Bell state measurement (BSM). We find that these approaches not only differ in the protocol efficiency but, remarkably, also in the teleportation fidelity, by removing unwanted coincidences of distinguishable photons. Their performance can be further improved when moderate spectral filtering (twice the radiative linewidth) is applied. To show the relevance of our approach, we present our results using a below-par $Q D$, i.e., one whose figures of merit (e.g., entanglement fidelity and photon indistinguishability, equal to 0.89 and 0.55 respectively) are below the average value found on the same sample. In this case, the average teleportation fidelity can be brought from below the classical limit-that is, failure of the protocol-to values as high as 0.84 . The experimental results are supported by a detailed theoretical model that explains how the imperfections of the source can be mitigated by the choice of the protocol. Our work thus shows that the search for the perfect $Q D$ can be relaxed and that the current quality of state-of-the-art QD entangled-photon sources is not so far from the more stringent requirements set by secure quantum communication applications ${ }^{18}$.

\section{RESULTS}

Source and setup overview

Among the various available materials systems, our choice for the source fell on GaAs quantum dots grown by droplet etching epitaxy. The major advantage of this technology is the capacity to generate highly entangled states ${ }^{19,20}$, up to 0.98 fidelity to a Bell state $^{21}$. The emission wavelength of emission can be designed within the high-efficiency spectral region of femtosecond Ti: sapphire lasers and silicon-based avalanche photodiodes and finetuned with strain or external fields to match Rb-based slow-light media $^{22-24}$ and quantum memories ${ }^{25,26}$. While, on the one hand,

\footnotetext{
${ }^{1}$ Department of Physics, Sapienza University of Rome, 00185 Rome, Italy. ${ }^{2}$ Department of Applied Physics, Royal Institute of Technology, 10691 Stockholm, Sweden. ${ }^{3}$ Institute of Semiconductor and Solid State Physics, Johannes Kepler University, 4040 Linz, Austria. ${ }^{4}$ Department of Physics, Paderborn University, 33098 Paderborn, Germany.

凶email: francesco.bassobasset@uniroma1.it; rinaldo.trotta@uniroma1.it
} 

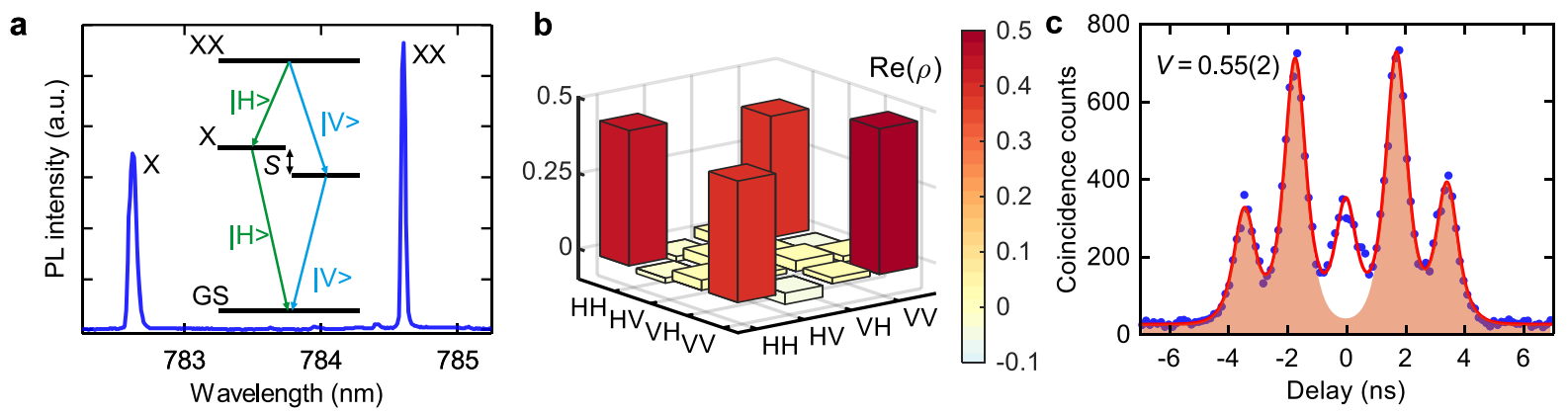

Fig. 1 Main properties of the investigated QD. a Photoluminescence spectrum of the investigated QD under resonant two-photon excitation of the $X X$ state. In the central inset, the radiative cascade process is sketched with its energy diagram. $\mathbf{b}$ Real part of the experimental density matrix which describes the polarization state generated via the XX-X cascade in the rectilinear basis. c Intensity correlation histogram (blue dots) recorded in a HOM experiment on X photons with a relative emission delay of $1.8 \mathrm{~ns}$. The data are fitted with the sum of five Gaussian functions convoluted to an exponential decay (red line), sharing the same FWHM. The fitted area of the side peaks is highlighted. The estimated interference visibility is also reported.

we make use of a state-of-the-art epitaxial technique, on the other hand, we do not resort to any strategy based on sample processing, external tuning, or performance-based selection to compensate for the unavoidable variability in the bottom-up fabrication process ${ }^{27-29}$. Therefore, we tackle the issue of source imperfections by considering a QD with suboptimal characteristics.

Figure 1a shows the emission spectrum of the QD studied in this paper, which contains the signature of the two optical transitions of the biexciton-exciton (XX-X) radiative cascade. Using a resonant two-photon excitation scheme, the photon pairs are produced with a preparation fidelity of 0.88 , as estimated by intensity cross-correlation measurements ${ }^{30}$. To show how the protocol works with suboptimal QDs, we chose one with a linewidth far larger than the average value of the sample. The FWHM of the $X$ and XX line is 118 and $68 \mu \mathrm{eV}$ respectively. We attribute this to a particularly noisy QD environment that causes spectral diffusion at both short and long timescales ${ }^{31}$. We notice that the $X$ line is broader than the $X X$ line (a commonly observed feature for $\mathrm{GaAs} \mathrm{QDs}^{31,32}$ ), but the difference in integrated intensity is less than $3 \%$, suggesting that the role of $\mathrm{XX}$ relaxation processes alternative to the $\mathrm{XX}-\mathrm{X}$ radiative cascade is negligible. The single-photon count rate is slightly above $1.5 \mathrm{MHz}$ for a $320 \mathrm{MHz}$ effective rate of laser pulses.

The features of the entangled photon source which are more relevant to the performance of the quantum teleportation operation are the degree of entanglement and photon indistinguishability ${ }^{30}$. In Fig. $1 \mathrm{~b}$ we report the density matrix that describes the polarization state of the photons emitted through the $\mathrm{XX}-\mathrm{X}$ radiative cascade. A liquid crystal retarder was set to maximize the fidelity to the state $\left|\phi^{+}\right\rangle=(|H H\rangle+|V V\rangle) / \sqrt{2}$, resulting in a matrix with no significant imaginary part (no matrix element above 0.03 in absolute value). The procedure is discussed in detail in the Supplementary Note 2. The fidelity to the Bell state $\left|\phi^{+}\right\rangle$is $0.89(1)$ and the concurrence of the matrix is $0.79(1)$, values well below the 0.98 and 0.97 that can be found in this materials system $^{21}$. This is due to the presence of a finite fine structure splitting (FSS) between the bright exciton levels, which is estimated to be 1.8(5) $\mu \mathrm{eV}$. Next, we assess the photon indistinguishability of the $X$ photons, which undergo the BSM in our teleportation scheme (see below), by performing two-photon interference in a Hong-Ou-Mandel (HOM) experiment. We select a single bright exciton component using a linear polarizer, so that the measurement is not influenced by the finite FSS. From the data reported and fitted in Fig. 1c, we estimate a visibility $V=0.55(2)$. This figure is lower than the value of approximately 0.65 commonly reported for GaAs QDs with similar characteristics ${ }^{16}$. While the main reason for below-unity visibility is arguably the time correlation between photons emitted in a cascaded

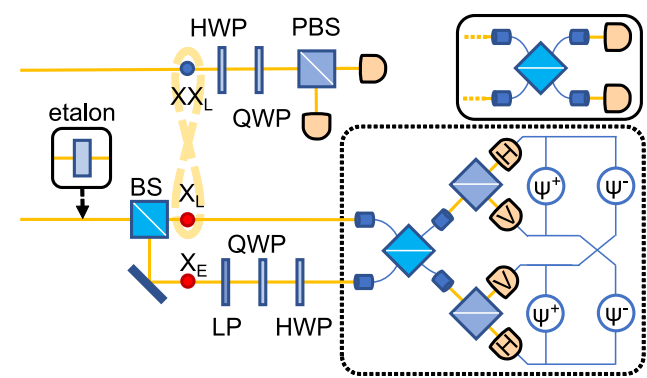

Fig. 2 Quantum teleportation using a QD photon emitter. Schematic illustration of the quantum teleportation setup. The BSM is implemented as shown in the dashed box, with a nonpolarizing beam splitter (BS) followed by two polarizing beam splitters (PBS) at its output ports, allowing for the detection of two different Bell states. In the solid boxes, we highlight the setup variations compared in this work. The detectors on the same side of the BS can be grouped to simulate the simpler procedure without polarization-selective elements. The $X$ photons can be filtered with an etalon whose bandwidth is approximately twice the radiative linewidth of the QD emission.

process $^{33-35}$, in this case a contribution from environmental field fluctuations is also probable, given the notable level of spectral diffusion in the time-integrated spectrum. Instead, we can safely assume no major reduction comes from the multi-photon emission of the source, because of the low values of the second-order autocorrelation function at zero-time delay $g_{\mathrm{x}}^{(2)}(0)=$ $0.011(1)$ and $g_{\mathrm{xx}}^{(2)}(0)=0.020(1)$. The deviation from zero is mainly attributed to the incomplete suppression of the laser backscattering and to the afterpulsing effect of the detectors ${ }^{36}$. Having described the main features of our source, we now move to the experimental setup.

We build upon the quantum teleportation protocol employed in one of the seminal experimental demonstrations ${ }^{2}$. A qubit is initially encoded in the polarization state of a photon. This photon, together with one from a polarizationentangled pair, is projected with a measurement onto a Bell state, in such a way that its initial polarization state is transferred to the remaining photon of the entangled pair, after a unitary transformation which depends on the specific outcome of the BSM. Here, we specifically focus on the actual implementation of the BSM and improve on the basic setup employed in the recent realization of all-photonic quantum teleportation with deterministic QD-based sources ${ }^{13}$.

Figure 2 summarizes the crucial parts of the setup. The protocol is performed using two pairs of entangled photons 
a

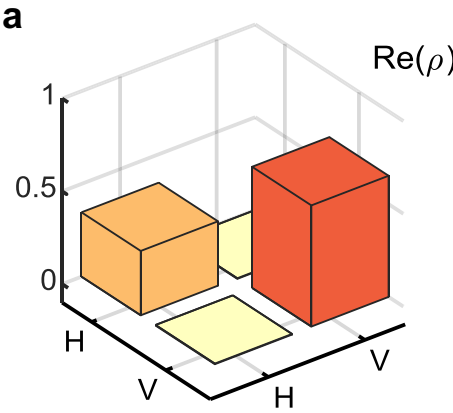

d

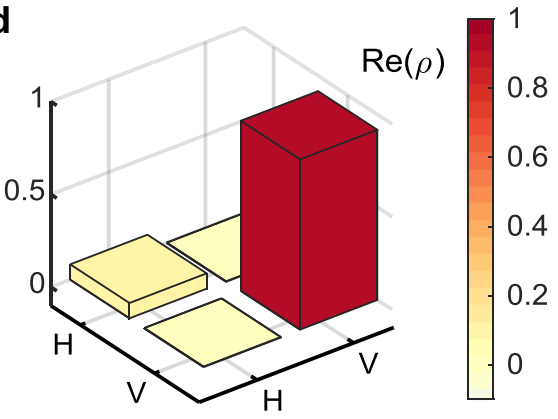

b

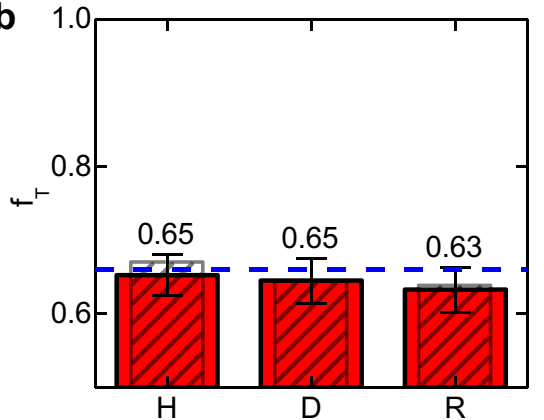

e

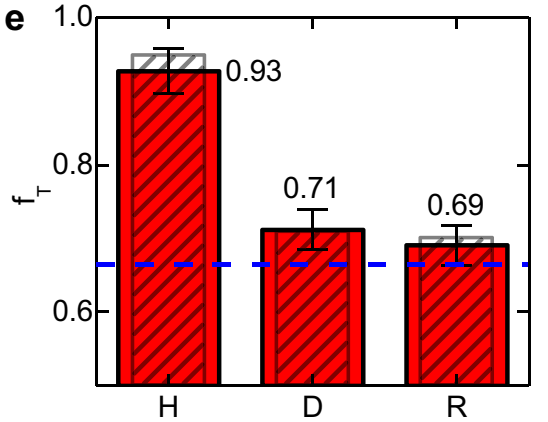

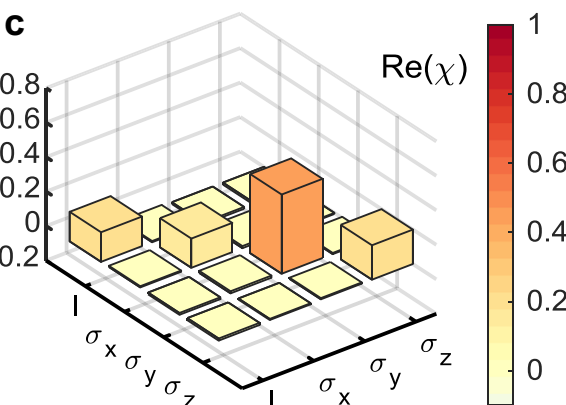

0.8

0.6

0.4

0.2

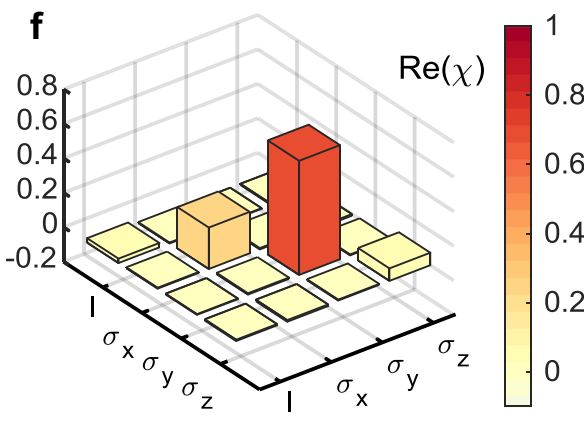

Fig. 3 Quantum teleportation with different BSM implementations. a, b, c Measurement of the $\left|\psi^{-}\right\rangle$state with a balanced non-polarizing beam splitter alone. In (a) density matrix of the teleported state corresponding to the input polarization state $\mathrm{H}$. In (b) teleportation fidelities for the $H, D$, and R input polarization states. The red filled bars represent the experimental values, including error bars (s.d.), the grey striped patterned bars are the values simulated based on the physical properties of the source. The blue dashed line represents the classical limit. In (c) quantum process tomography matrix. d, e, f Same as panels (a), (b), and (c) respectively, in the case in which the measurement of the $\left|\psi^{-}\right\rangle$ state includes polarizing beam splitters in the setup.

emitted by the same QD in two independent excitation events related to consecutive laser pulses, which we name early (E) and late $(L)$ in their respective order. From the early photon pair, we only consider the photon from the $X$ to ground state transition -namely the $X_{E}$ photon-which serves as the input state of the protocol. Its polarization is set using a linear polarizer (LP) followed by either a half-wave plate (HWP) or a quarter-wave plate (QWP). The late photon pair is spectrally split so that the $X X_{L}$ photon is sent to a polarizing beam splitter preceded by a QWP and a HWP, to perform quantum state tomography on the outcome of the teleportation protocol. The $X_{L}$ photon undergoes a BSM together with $X_{E}$. Its easiest implementation, shown in the upper right box of Fig. 2, relies on a non-polarizing beam splitter to observe two-photon interference. This allows for detecting only the antisymmetric Bell state, namely $\left|\psi^{-}\right\rangle=(|H V\rangle-|V H\rangle) / \sqrt{2}$, which caps the success rate of the protocol at $25 \%$. The problem of how to realize a complete BSM has no easy solution ${ }^{37,38}$, but the performance of the protocol can be improved with common linear optical elements ${ }^{39,40}$. The approach consists of adding two polarizing beam splitters at the output ports of the non-polarizing beam splitter, as illustrated in the main part of Fig. 2, and detecting photons from four channels instead of two. Considering pairs of detectors at the outputs of the same polarizing beam splitter, it is possible to measure the Bell state $\left|\psi^{+}\right\rangle=(|H V\rangle+|V H\rangle) / \sqrt{2}$ as well. At this point, one could naively think that these modifications only increase the success rate of the teleportation protocol to $50 \%$, but this is not the whole picture when imperfections of the source are considered. In fact, the BSM setup with $50 \%$ discrimination probability does not only use photon indistinguishability to distinguish different Bell states, but also information about the polarization state. We will discuss and quantify this difference while presenting the results obtained with the different setups.

Optimization by polarization-selective Bell state measurement First, we report the results of the quantum teleportation operation when the polarizing beam splitters in the BSM are disregarded, effectively mimicking the minimal setup with $25 \%$ efficiency sketched in the upper right box of Fig. 2.

Together with the coincident click of $X$ photons exiting the nonpolarizing beam splitter at different output ports within a time window of $0.6 \mathrm{~ns}$, we record the polarization state of the $X X_{L}$ photon. For each input state, we measure the teleported state in the horizontal, diagonal, and circular polarization bases. In this way, the polarization density matrix is derived from the Stokes parameters ${ }^{41}$. The total rate of threefold coincidences is $0.8 \mathrm{~Hz}$, which is expected from the efficiency figures of merit of the setup and the source ${ }^{30}$. In Fig. 3a we report the output obtained when the input photon $X_{E}$ is sent through a linear polarizer oriented in the lab horizontal direction, selecting the $\mathrm{H}$ state. The first eigenvector is equal to its orthogonal state $\mathrm{V}$, which is the expected outcome of the quantum teleportation operation when $\left|\psi^{-}\right\rangle$is the measured Bell state, that is a bit-phase flip described by the Pauli matrix $\sigma_{y}$. The overall state is mixed and the fidelity to the $\mathrm{V}$ state is limited to $0.65(3)$. Figure $3 \mathrm{~b}$ reports the teleportation fidelity for the diagonal (D) and right-circular (R) states as well, a set that allows evaluating the average gate fidelity for an arbitrary input $^{10}$, if the degree of polarization of the source is null. The average fidelity is equal to $0.644(17)$ and does not surpass the classical limit of $2 / 3$, which corresponds to the threshold accuracy for the faithful cloning of a qubit ${ }^{42}$. This conclusion is consistent with the source requirements set in previous works adopting the same BSM approach ${ }^{13}$. With an additional set of measurements we are also able to reconstruct the quantum process matrix ${ }^{43}$ of 
the teleportation protocol. The result shown in Fig. $3 \mathrm{c}$ is a diagonal matrix whose elements correspond to the basic quantum operations $I, \sigma_{x}, \sigma_{y}, \sigma_{z}$, namely the Pauli matrices. These operations describe how the input state transfers to the output states in the ideal quantum teleportation for different outcomes of the BSM, $\left|\phi^{+}\right\rangle,\left|\psi^{+}\right\rangle,\left|\psi^{-}\right\rangle$, and $\left|\phi^{-}\right\rangle$respectively. The process in Fig. $3 \mathrm{c}$ is essentially a mixed state of the Pauli matrix $\sigma_{y}$-the ideal operation for a $\left|\psi^{-}\right\rangle$measurement, to which our matrix retains a 0.46 fidelity-together with the matrices related to the other BSM outcomes, present in similar proportions. From the quantum process matrix the average gate fidelity can be derived in a straightforward manner ${ }^{44}$, yielding a value of 0.64 , consistent with the estimate from Fig. $3 \mathrm{~b}$ and still below the classical limit.

As mentioned above, the fidelity is expected to improve when we move to the polarization-selective BSM, i.e., when we consider the detectors corresponding to orthogonal linear polarization states as counting for a projection onto the Bell state $\left|\psi^{-}\right\rangle$. The difference is most striking for the input state $\mathrm{H}$, as displayed in Fig. $3 \mathrm{~d}$. The purity of the output state is substantially increased, with a fidelity to $V$ which is now as high as $0.93(3)$. The enhanced protocol also allows extending the operation to the detection of $X$ photons in the Bell state $\left|\psi^{+}\right\rangle$. For each investigated input state, we observe that the discrepancy between the teleportation fidelities based on $\left|\psi^{+}\right\rangle$and $\left|\psi^{-}\right\rangle$detection lies within the statistical error (see the Supplementary Fig. 2 for the complete comparison). In Fig. 3e we report the teleportation fidelities for different input states on the Poincaré sphere averaged over the different possible combinations of detection channels. The overall average teleportation fidelity is $0.776(16)$, more than six standard deviations above the classical limit. While we will discuss these results more quantitatively below, the quantum process matrix tomography for the measurement of the Bell state $\left|\psi^{-}\right\rangle$in Fig. 3f already offers an intuitive picture of the main advantage of a polarization-selective BSM. Since coincidence events from distinguishable linearly co-polarized photons are discarded, teleportation events linked to the projection of $\mathrm{X}$ photons onto the Bell states $\left|\phi^{+}\right\rangle$and $\left|\phi^{-}\right\rangle$are removed. This can be noticed from the strong decrease in the matrix elements relative to the identity and to the Pauli matrix $\sigma_{z}$ with respect to the matrix of Fig. 3c. The increased purity of the process results in an average gate fidelity of 0.78 and 0.76 for the $\left|\psi^{-}\right\rangle$and $\left|\psi^{+}\right\rangle$case respectively (see the Supplementary Fig. 3 for the matrix comparison).

Despite the strong improvement, we can object from Fig. 3e that the teleportation fidelity is still very close to the classical limit when sending $D$ and $R$ polarizations. Indeed, classical correlation and sorting of the polarization states of the $X$ photons in the horizontal basis are sufficient to transfer the states $\mathrm{H}$ and $\mathrm{V}$. Entanglement and a selective BSM are instead essential to guarantee the protocol success regardless of the input state. The main limitation for reliably sending $D$ and $R$ states is set by the limited photon indistinguishability of $X$ photons.

\section{Optimization by spectral filtering}

Photon indistinguishability can be improved by means of spectral $^{45}$ or temporal ${ }^{46}$ post-selection. Here, we study the effect of an air-spaced etalon with a bandwidth of $1.28 \mathrm{GHz}$, corresponding to $5.47 \mu \mathrm{eV}$. The introduction of this element obviously comes with a tradeoff in terms of source brightness. Given the large spectral diffusion of the emitter of this case study, this reduction amounts to a factor 10 on the $\mathrm{X}$ emission line after filtering. As we experimentally measured, this corresponds to a factor 35 decrease in the threefold coincidences. This value is lower than the factor 100 expected for uncorrelated losses. If one $X$ photon gets transmitted by the etalon filter, the conditional probability that a second one, emitted after $1.8 \mathrm{~ns}$, gets transmitted as well is higher than $1 / 10$, because most spectral wandering effects take place on longer timescales.
The losses just reported refer, though, to an unfavorable case study, where our focus is more on the success probability of the protocol rather than on its throughput. It should be noted that the filter considered here is twice as large as the radiative linewidth of the $X$ transition, so that the loss can be greatly reduced when investigating QDs with linewidth closer to the Fourier limit. An intensity loss down to a factor 4 was indeed measured for other emitters in the same sample. QDs in a sample grown with the same materials and epitaxial technique have displayed linewidths only a factor two above the Fourier limit ${ }^{16}$, for which we could expect the intensity to be halved when filtered by the etalon used in this experiment. In this latter realistic sample, the reduction in three-fold coincidences could reasonably be expected to be a factor 4, even assuming uncorrelated losses.

Despite the modest selectivity of our filtering approach, its addition is greatly effective. The HOM visibility of $X$ photons coming from consecutive radiative cascades is increased from 0.55 (2) to $0.79(2)$ as displayed in Fig. 4a. This value is higher than the typical ones reported for similar GaAs QDs with the same excitation scheme and an $X$ linewidth twice the Fourier limit ${ }^{16}$, suggesting that the etalon effect is not limited to removing spectral wandering. As previously mentioned, the main limiting factor for photon indistinguishability is arguably the time correlation between photons emitted in a cascaded process ${ }^{33,34}$. From the point of view of the $X$ photons, that is tracing out the information on the XX photons, this could be more intuitively seen-with some simplification ${ }^{35}$ - as an effective time jittering due to the fact that a random time passes before the system decays from the $X X$ to the $X$ state. Now, the introduction of the etalon filter also has an impact on the wave packet of the emitted photons. From the timedependent photoluminescence illustrated in Fig. $4 \mathrm{~b}$ we can infer that the temporal width of the photon wave function is broadened because of the spectral filtering. The effective temporal jittering with which the $X$ state is populated is unchanged, but it appears smaller relatively to the increased temporal width of the photon wave packet. Since this mechanism would increase the overlap of the photon wave functions at the beam splitter, it could provide an explanation for the improvement of the two-photon interference visibility. Nonetheless, further detailed analysis of the effect is left to future studies. Here, it is only important to notice that the change in the HOM visibility is not accompanied by a modification of either the photon emission statistics, with $g_{\mathrm{X}}^{(2)}(0)=0.009(1)$, or the degree of entanglement of the source, with a fidelity to the Bell state $\left|\psi^{-}\right\rangle$of $0.90(1)$.

We repeated the teleportation experiment including the etalon filter at the entrance of the BSM apparatus. As expected, the higher HOM visibility of the $X$ photons directly improves the success rate of the protocol. The results are reported in Fig. 4c. The teleportation fidelity relative to the input state $\mathrm{H}$ is essentially unchanged, whereas the values for the $D$ and $R$ input states are significantly increased with respect to the case of Fig. 3e and are now safely above the classical limit as well. This results in an average teleportation fidelity increased to $0.842(14)$, as evident from the comparison in Fig. $4 d$.

\section{DISCUSSION}

In order to place the results reported so far into a quantitative framework, we introduce a formal description of the quantum teleportation operation for an imperfect entangled photon source. The protocol starts from the three-photon state $\rho_{\psi}=|\Phi\rangle_{X_{E}}\left\langle\left.\Phi\right|_{X_{E}} \otimes \rho_{X_{L}, X_{L}}\right.$, where $\left.\mid \Phi_{X_{E}}\right\rangle$ is the input pure state to be transferred, and $\rho_{X_{L}, X X_{\llcorner}}$is the density matrix representation of the polarization state of the photon pair emitted by the XX-X cascade. The latter matrix describes, in general, a mixed state, which can either be experimentally measured or simulated from the relevant figures of merit of the source. Following the model of Hudson et al. ${ }^{47}$, the relevant properties are FSS $S$, multiphoton 


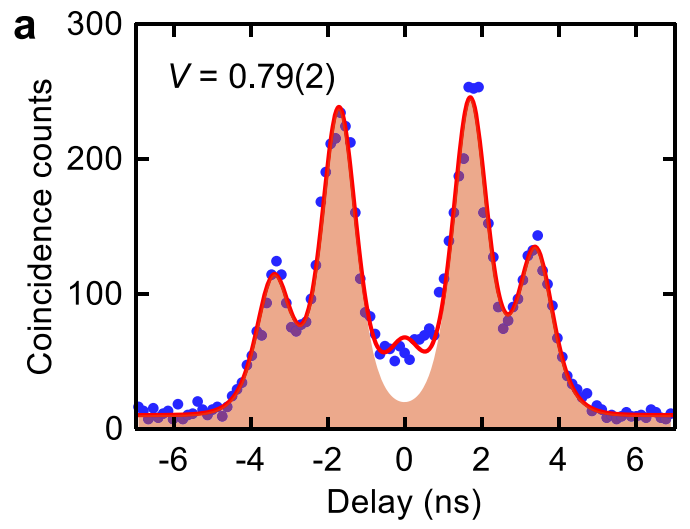

b
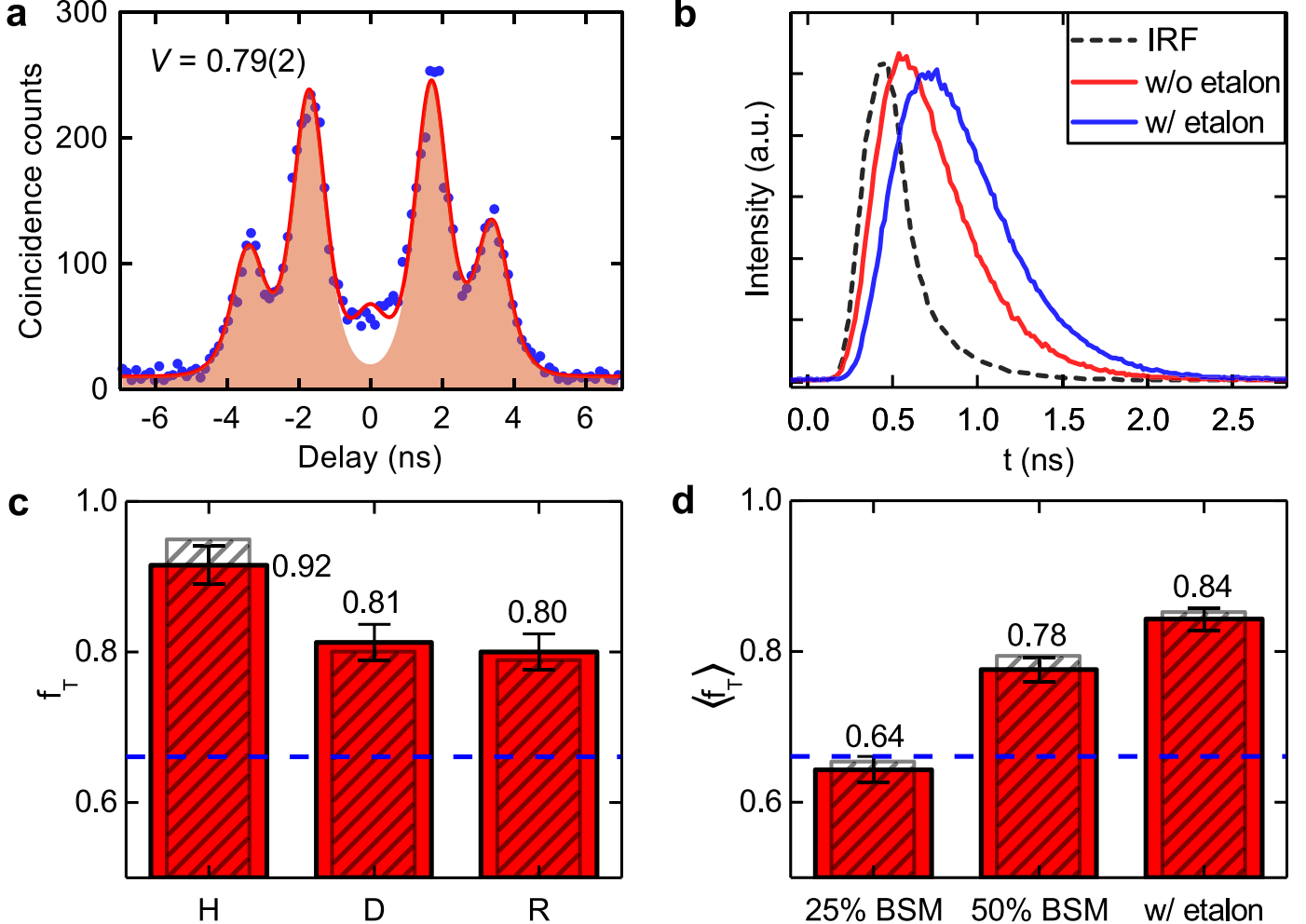

Fig. 4 Quantum teleportation with spectral filtering. Measurements performed on $\mathrm{X}$ photons transmitted through a $1.28 \mathrm{GHz}$ bandwidth etalon. a Intensity correlation histogram (blue dots) recorded in a HOM experiment as in Fig. 1c. The data are fitted with the sum of five Gaussian functions convoluted to an exponential decay (red line), sharing the same FWHM. The fitted area of the side peaks is highlighted. The estimated interference visibility is also reported. b Time-resolved photoluminescence decay curves. Comparison between the case without (red curve) and with (blue curve) the presence of the etalon. The black dashed curve represents the instrument response function (IRF), which is convoluted with an exponential decay to fit the red curve and deduce the total lifetime of the $\mathrm{X}$ to ground state transition. c Teleportation fidelities for the H, D, and R input polarization states. The red filled bars represent the experimental values, including error bars (s.d.), the grey striped patterned bars are the values simulated based on the physical properties of the source. The blue dashed line represents the classical limit. d Comparison of the average teleportation fidelity for the three approaches to quantum teleportation studied in this work.

emission, and characteristic times of decoherence between the bright $X$ states, namely the spin scattering time $\tau_{\mathrm{sS}}$, which describes physical mechanisms that erase any polarization correlation, and the cross-dephasing time $\tau_{\mathrm{HV}}$, which relates to events in which the phase coherence between the two bright exciton states is lost. The teleportation protocol acts on the three-photon state as a projection of the $X$ photons onto a specific Bell state, e.g., $\left|\psi^{-}\right\rangle$, followed by a partial trace to return the teleported state:

$\rho_{\mathrm{XX}}^{\psi_{\mathrm{L}}^{ \pm}}=\sum_{i=\phi^{+}, \phi^{-}, \psi^{+}, \psi^{-}} p\left(i \mid B S M_{\psi^{ \pm}}\right) \operatorname{Tr}_{\mathrm{X}_{\mathrm{E}}, \mathrm{X}_{\mathrm{L}}}\left[\frac{\Pi_{\mathrm{X}_{\mathrm{E}}, \mathrm{X}_{\mathrm{L}}}^{\mathrm{i}} \rho_{\psi} \Pi_{\mathrm{X}_{\mathrm{E}}, \mathrm{X}_{\mathrm{L}}}^{\mathrm{i}}}{N^{i}}\right]$

where $\Pi_{\mathrm{X}_{\mathrm{E}}, \mathrm{X}_{L}}^{\mathrm{i}}$ is a projection operator onto the Bell state $i, N^{i}$ is a normalization factor, and $p\left(i \mid B S M_{\psi^{ \pm}}\right)$is the probability that a Bell state detection event, here labeled as $B S M_{\psi^{ \pm}}$, is actually triggered by a photon pair in the Bell state $i$. The latter term accounts for inaccuracies in the BSM stemming from the non-ideal HOM visibility and FSS and, together with the expression for $\rho_{\psi}$, describes the influence of QD imperfections on the teleported state. To make this point clear, consider the example in which two $X$ photons are recorded at the separate sides of the nonpolarizing beam splitter, and we tag the corresponding teleportation event as triggered by the detection of the Bell state $\left|\psi^{-}\right\rangle$. If the $\mathrm{X}$ photons are not fully indistinguishable, it is possible though that also photon pairs with a symmetric polarization state exit separately from the beam splitter. These accidental events are quantified in the non-zero values of $p\left(\psi^{+} \mid B S M_{\psi^{-}}\right), p\left(\phi^{-} \mid B S M_{\psi^{-}}\right), p\left(\phi^{+} \mid B S M_{\psi^{-}}\right)$that, via the sum in
Eq. (1), enter in the final teleported state. It is also important to point out that $p\left(i \mid B S M_{\psi^{ \pm}}\right)$is exactly the term that changes for the different setup implementations investigated in this work. Therefore, it is worth taking a look at how it depends on the $\mathrm{HOM}$ visibility and the $\mathrm{FSS}^{30}$. The dependence on the HOM visibility is summarized in Fig. $5 \mathrm{a}$, b for the cases of the BSM with $25 \%$ and $50 \%$ efficiency respectively (see the Supplementary Note 3 for the detailed formulas). Comparing the two graphs immediately highlights how the model treats different setup implementations of a $\left|\psi^{-}\right\rangle$BSM. The inclusion of the polarizing beam splitters allows discarding with high precision coincidence events from linearly co-polarized photons, which means that the probability that a potential teleportation event is caused by $\mathrm{X}$ photons in a $\left|\phi^{+}\right\rangle$or $\left|\phi^{-}\right\rangle$state is negligible. This results in a dramatically increased accuracy of the BSM, especially when considering interference between photons with poor indistinguishability. It is worth noting that, even in the limit of HOM visibility equal to unity, the BSM can have below unity accuracy. The HOM visibility accounts for several effects such as dephasing, spectral wandering and time jittering, but is measured on a single bright exciton component and, therefore, does not include the effect of FSS on the photon indistinguishability. Even if the single bright exciton state is populated with no time jittering and its line is Fourier limited, a $\left|\psi^{+}\right\rangle$state has a non-vanishing probability of being tagged as $\left|\psi^{-}\right\rangle$in the BSM, since linearly cross-polarized photons are partially distinguishable if a frequency detuning due to FSS is present. This scenario is represented by the solid curve in Fig. $5 a, b$ for the case in which the FSS is fixed to the value of 1.8(5) $\mu \mathrm{eV}$ of the investigated QD. 
a

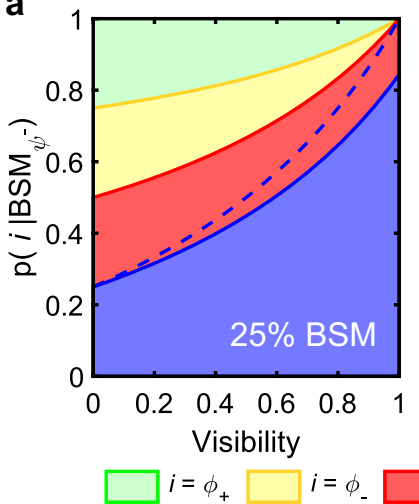

b

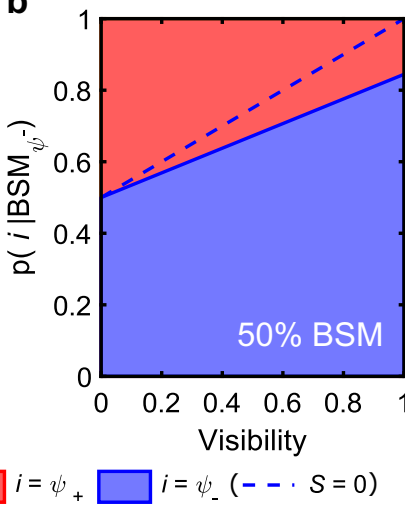

c

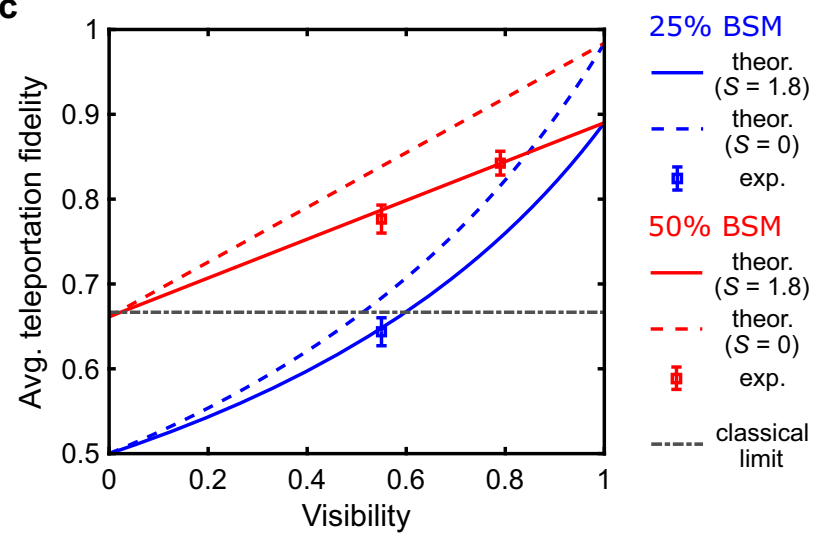

Fig. 5 Modeling with realistic source and setup. a Probabilities that a BSM is caused by two photons in a given Bell state for the $25 \%$ efficiency setup, where the detectors at the same output of the non-polarizing beam splitter are grouped together. The probabilities are displayed in a stacked area plot as a function of the HOM visibility and are calculated both with all the physical properties of the investigated QD (solid curve, $S=1.8 \mathrm{eV}$ ) and assuming zero FSS (dashed curve). b Same as panel (a) but focusing on the BSM of the state $\left|\psi^{-}\right\rangle$in the $50 \%$ efficiency setup. Identical results apply to the state $\left|\psi^{+}\right\rangle$by simply swapping the labels between the two states. c Simulated values of the average teleportation fidelity as a function of the HOM visibility for both the implementations of the BSM. Calculated both with all the physical properties of the investigated QD (solid curve, $S=1.8 \mu \mathrm{eV}$ ) and assuming zero FSS (dashed curve). The graph also presents the experimental average fidelities (square dots), including error bars (s.d.), from Fig. 4d for comparison.

We can evaluate these conditional probabilities for the QD of interest from a few measurable physical properties of the source, namely HOM visibility, FSS, $X$ radiative lifetime and pure dephasing rate $T_{2}{ }^{*}$. It is worth noting that the latter parameter is not used to estimate photon indistinguishability, since its contribution is already empirically included in the value of HOM visibility. In fact, $T_{2}{ }^{*}$ is only used in the model to better quantify the impact of FSS on the BSM accuracy, since the effect of FSS is not included in the HOM visibility. As such, the precise estimation of the dephasing rate (for photons emitted with $1.8 \mathrm{~ns}$ relative delay) only marginally affects the predictions of the model, and we adopt a literature value from a comparable GaAs $\mathrm{QD}^{31}$.

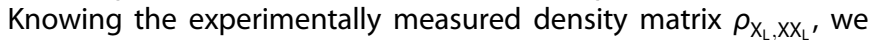
are able to simulate the teleported state for each experimental condition and input polarization analyzed in our work. The teleportation fidelities relative to the simulations are indicated by grey striped bars in Figs $3 b, e, 4 c$, $d$. The excellent agreement with the experimental data fully supports our previous insights on the role of the polarization-selective BSM and the spectral filtering on the success rate of the quantum teleportation protocol based on photons from a quantum emitter.

The model also qualifies as a useful tool in guiding the design of a source useable in a realistic quantum communication protocol. By including a formula for the entangled pair density matrix as well, it is possible to predict the protocol performance from a few physical properties of the source alone. We add a minor variation with respect to the matrix introduced by Hudson et al. ${ }^{47}$ (see the Supplementary Note 2 for the complete expression), by also accounting for a unitary transformation applied on both photons to maximize the fidelity to the Bell state $\left|\phi^{+}\right\rangle$, which is done in our setup with a liquid crystal retarder.

In this way, we obtain an analytical expression for the quantum process matrix $X$ which provides an exhaustive description of the teleportation procedure ${ }^{43}$. $X$ is a diagonal matrix, whose expression depends on the specific outcome of the BSM, in this case, either $\left|\psi^{-}\right\rangle$or $\left|\psi^{+}\right\rangle$:

$\chi_{00}^{\psi^{ \pm}}=X_{33}^{\psi^{ \pm}}=\frac{1-c^{\prime} k g_{\mathrm{H}, \mathrm{V}}^{\prime(1)}}{4}$

$X_{11}^{\psi^{ \pm}}=X_{22}^{\psi^{\mp}}=\frac{1}{4}\left(1+c^{\prime} k g_{\mathrm{H}, \mathrm{V}}^{(1)} \pm c \frac{2 k g_{\mathrm{H}, \mathrm{V}}^{(1)}}{\sqrt{1+\left(\frac{S \tau_{0}}{\hbar} g_{\mathrm{H}, \mathrm{V}}^{(1)}\right)^{2}}\left(1+\left(\frac{S \tau_{0}}{\hbar} g_{\mathrm{deph}}^{(1)}\right)^{2}\right)}\right)$

where $k$ is the fraction of detected photons pairs in which both photons come from the $\mathrm{XX}-\mathrm{X}$ cascade and not from multiphoton or background emission, $S$ is the FSS, $g_{H . V}^{\prime(1)}=1 /\left(1+\tau_{X} / \tau_{S S}\right)$, $g_{\mathrm{H}, \mathrm{V}}^{(1)}=1 /\left(1+\tau_{\mathrm{X}} / \tau_{\mathrm{SS}}+\tau_{\mathrm{X}} / \tau_{\mathrm{HV}}\right)$, and $g_{\mathrm{deph}}^{(1)}=1 /\left(1+2 \tau_{\mathrm{X}} / T_{2}^{*}\right)$. The coefficients $c$ and $c^{\prime}$ depend on the specific implementation of the $B S M$, either with (BS) or without (PBS) polarization-selective elements:

$c_{\mathrm{BS}}^{\prime}=\frac{V}{2-V}, c_{\mathrm{PBS}}^{\prime}=1$,

$c_{\mathrm{BS}}=\frac{V}{2-V}, c_{\mathrm{PBS}}=V$.

The FSS causes quantum teleportation to act as a mixture between $\sigma_{\mathrm{x}}$ and $\sigma_{\mathrm{y}}$ single-qubit gates, assuming that the symmetry axes of the QD are aligned with the horizontal polarization basis of the lab. Its impact is reduced by using a polarization-selective BSM except in the case of otherwise perfect photon indistinguishability. The effect of the BSM implementation is more drastic the lower the HOM visibility of the emitter. If we only rely on photon indistinguishability to sort out the Bell state, an imperfect overlap of the wave functions causes the ideal process matrix to mix with an identity matrix, the same effect as spin scattering mechanisms that erase any correlation in the polarization of the entangled photon pair. If we also select Bell states with the help of polarizing elements, the effect is limited to mixing the $\sigma_{x}$ and $\sigma_{y}$ elements of the process matrix, as for FSS and cross-dephasing mechanisms, and not to the elements related to the discarded $\left|\phi^{+}\right\rangle$and $\left|\phi^{-}\right\rangle$states.

From the quantum process tomography an expression for the average fidelity of teleportation can be easily obtained as well. In Fig. 5c, we show how the fidelity of teleportation changes with respect to the HOM visibility for both the QD investigated here and a similar one with its FSS brought to zero. The curves are compared once again with the experimental data, confirming the excellent agreement.

The prediction of the model in Fig. $5 \mathrm{c}$ gives us a tool to relate the figures of merit of the source with the performance requirements set by the desired application. In a quantum communication perspective, the quantum teleportation protocol can enable safe qubit exchange over a distance in a relayed mode. Notably, in order to perform quantum key distribution, the 
teleportation fidelity must be high enough not to exceed the maximum level of quantum bit error rate tolerated by the specific protocol. Using an error-resistant scheme, raising the threshold up to $27.6 \%$, the results of our work would already satisfy the requirement. Most commonly used protocols work with an $11 \%$ error threshold ${ }^{49}$, which is still a very realistic goal according to the prospects of our study.

In summary, we have investigated in detail how imperfections in a QD source affect the performance of quantum teleportation using photons and their polarization state. The FSS and, even more so, two-photon interference visibility may not be optimal in as-grown QDs and influence the probability of success of the protocol. However, their impact on the fidelity of the operation strongly depends on the implementation of the BSM in the setup. A direct comparison by means of quantum process tomography highlights that the inclusion of polarizing beam splitters, in addition to increasing the efficiency of the protocol, allows sorting out Bell states with higher accuracy and lowers the source requirements for quantum teleportation. This approach has also been studied in combination with spectral filtering. The choice of a relatively large bandwidth-double the radiative linewidthleads to a significant increase of the HOM visibility, in the test case from $0.55(2)$ to $0.79(2)$, while not affecting in a relevant way other important features such as photon statistics and degree of entanglement. Altogether, the average teleportation fidelity is raised from below the classical limit to $0.842(14)$. It should be acknowledged that the use of spectral filtering comes with a trade-off with respect to the teleportation rate, and this remains a downside for emitters with poor optical quality. In our specific case study three-fold coincidences are reduced by a factor 35 , which could increase to 100 for teleportation protocols involving remote emitters. Using realistic QDs with the exact same design and linewidth twice the Fourier limit ${ }^{16}$ we would already expect the reduction in teleportation events to be limited to a factor 4 for communication protocols with remote emitters, and even less for schemes relying on a single emitter as in quantum photonic simulators ${ }^{50}$. While waiting for the perfect Fourier-limited entangled photon source, spectral filtering can be the lesser of the evils compared to temporal filtering, when the emitter lifetimes are short with respect to the detector time resolution or when interacting with other systems-such as atomic vapor clouds $^{51}$ — with specific bandwidth requirements. Finally, we have presented and discussed a quantitative model able to predict the performance of the protocol from the physical properties of the source and the details of the setup, with the goal of providing a useful tool for experiment design. Our investigation could be easily extended to any generic polarization-entangled photon source relying on a radiative cascade emission process. The state of the art in this category is formed by a wide family of epitaxial QDs fabricated with different techniques and materials choices, that have already been proven viable for entangled photon generation ${ }^{52}$, together with neutral atoms ${ }^{53,54}$. In prospect, an even broader range of systems could be considered, because the development of polarization-entangled photons has been voiced as a realistic research goal for many other quantum emitters that are currently studied as single-photon sources: colloidal QDs ${ }^{55,56}$, molecules 57,58 , color centers in crystalline hosts ${ }^{59,60}$ or in $2 D^{\prime}$ materials ${ }^{61,62}$. Recent improvements in terms of sample fabrication and processing, such as integrated micromachined piezoactuators $^{27,51}$ and broadband photonic cavities ${ }^{63,64}$, hold great promise of tackling the limitations of existing quantum emitters at their roots. However, the emerging paths of development in the field-above all quantum communication over long distances ${ }^{12,65}$ and between remote emitters ${ }^{16}$ - come with additional technical challenges, so that managing source imperfections will remain a crucial task for reaching the next milestones. Therefore, we envisage that the strategies described in this work will be of utmost importance for the forthcoming construction of solidstate-based quantum networks.

\section{METHODS}

Entangled photon source

The polarization-entangled photons are generated by a single GaAs QD embedded in a crystalline matrix of $\mathrm{Al}_{0.4} \mathrm{Ga}_{0.6}$ As. The sample was fabricated on a GaAs (001) substrate using molecular beam epitaxy and the Al droplet etching technique ${ }^{66}$, as in other recent implementations of quantum communication protocols ${ }^{13,14}$. The growth parameters were chosen to minimize the degree of in-plane anisotropy of the nanostructures ${ }^{67}$. The QDs were positioned at the middle of a $123 \mathrm{~nm}$-thick layer of $\mathrm{Al}_{0.4} \mathrm{Ga}_{0.6} \mathrm{As}$ placed between two $60 \mathrm{~nm}$-thick layers of $\mathrm{Al}_{0.2} \mathrm{Ga}_{0.8} \mathrm{As}$, which together constitute a $\lambda$-cavity. This cavity was inserted in between two distributed Bragg reflectors, composed by alternating layers of $70 \mathrm{~nm}$ of $\mathrm{Al}_{0.95} \mathrm{Ga}_{0.05} \mathrm{As}$ and $60 \mathrm{~nm}$ of $\mathrm{Al}_{0.2} \mathrm{Ga}_{0.8} \mathrm{As}$. The bottom part was formed by 9 pairs of layers, whereas the upper part was formed by 2 pairs of layers to facilitate light collection from the top sample surface. The sample was capped by $4 \mathrm{~nm}$ of GaAs to avoid surface oxidation. Due to the Bragg reflectors and a half-ball lens made of N-LASF9 placed on top of the sample, an extraction efficiency of approximately $12 \%$ is achieved for emission wavelengths around $785 \mathrm{~nm}$.

\section{Quantum teleportation setup}

The entangled photon source operates at a temperature of $5 \mathrm{~K}$ in a lowvibration closed-cycle He cryostat from attocube systems. The QD is driven via a resonant two-photon excitation scheme ${ }^{68}$ employing a Ti:Sapphire femtosecond laser with an $80 \mathrm{MHz}$ repetition rate. The rate of laser pulses is effectively doubled using an unbalanced Mach-Zehnder interferometer with a delay of $6.25 \mathrm{~ns}$. Another similar interferometer creates consecutive laser pulses with $1.8 \mathrm{~ns}$ delay, as required for synchronous photon detection from a single source in the BSM and HOM setup. A $4 \mathrm{f}$ pulse shaper with an adjustable slit on its Fourier plane is used to reduce the linewidth of the laser down to approximately $200 \mu \mathrm{eV}$.

A 0.81 NA objective placed inside the cryostat focuses the laser on the sample and collects the signal from the QD. The backscattered light from the laser is removed by volume Bragg gratings notch filters with a bandwidth of $0.4 \mathrm{~nm}$. A second set of volume Bragg gratings allows us to efficiently single out the emission from each of the two transitions of the $\mathrm{XX}-\mathrm{X}$ cascade and reflect them into different parts of the setup. A liquid crystal retarder is used to compensate for any unitary transformation of the photon polarization induced by the optical elements, so that the twophoton polarization state retains maximal fidelity to the entangled state $\left|\phi^{+}\right\rangle$. Photons from the $\mathrm{X}$ to ground state transition are sent to the section of the setup devoted to the BSM. When specified, an air-spaced etalon with a bandwidth of $1.28 \mathrm{GHz}$ and a free spectral range of $15 \mathrm{GHz}$ is inserted before the BSM setup. The etalon is tilted in such a way that the signal transmission is maximal and equal for all its polarization components. An unbalanced Mach-Zehnder interferometer with a delay of $1.8 \mathrm{~ns}$ is inserted to match the delay introduced in the excitation path between consecutive laser pulses. Its second beam splitter is single-mode fiber-coupled with $48 \%$ reflectance, $52 \%$ transmittance, and a mode overlap of $96 \%$. Each of the outputs leads to a polarizing beam splitter. Two paddle (bat-ear) polarization controllers and a set of three zero-order waveplates, in the usual quarter-wave, half-wave, and quarter-wave retardance combination, are used to preserve any input polarization state through the single-mode fibers. Photons from the $X X$ to $X$ transition go through a zero-order waveplate, with either quarter-wave or half-wave retardance, and a polarizing beam splitter to perform single-qubit quantum state tomography. A silicon avalanche photodiode with a time jitter of approximately $400 \mathrm{ps}$ and a nominal quantum detection efficiency of $65 \%$ is placed at every output of each polarizing beam splitter-six in total. The singlephoton counts are recorded by time tagging electronics with $10 \mathrm{ps}$ resolution from Swabian Instruments and then analyzed to yield threefold coincidences histograms for different combinations of channels. A complete sketch of the experimental setup is reported in the Supplementary Methods.

The autocorrelation function of the $\mathrm{XX}$ and $\mathrm{X}$ emission, the $\mathrm{XX}-\mathrm{X}$ polarization density matrix, and the HOM visibility of $X$ photons from consecutive emission events are measured in the same setup. We estimate the HOM visibility from the ratio of the integrated intensity of the zerotime delay peak with respect to its side peaks in the measured coincidences histogram ${ }^{69}$. The peaks are modeled and fitted with five 
Gaussian functions convoluted with a symmetric exponential decaywhich represent the instrument response function and the lifetime of the emitter respectively - with fixed width and different intensities. The result of the fitting procedure on the data is directly used to evaluate the integrated intensity of the side peaks. The zero-time delay peak has, in general, a more complex functional form due to the presence of quantum beats $^{70}$, so that the fit function does not fully reproduce its features even when the quantum beats are hidden by the limited time resolution. We calculated the integrated intensity of the central peak from the experimental data directly after having subtracted the fitted area of the side peaks.

Another silicon avalanche photodiode with a time jitter slightly below 300 ps (FWHM) is used for lifetime measurements. The emission spectra of the $\mathrm{QD}$ are acquired by a deep-depletion, back-illuminated $\mathrm{LN}_{2}-\mathrm{CCD}$ camera using a $750 \mathrm{~mm}$ focal length spectrometer, equipped with an $1800 \mathrm{~g} / \mathrm{mm}$ grating.

\section{Data and error analysis}

The errors in the estimation of the experimental fidelities are calculated by Gaussian propagation assuming a Poissonian distribution of the count of threefold coincidence events.

During the teleportation experiments, the data is recorded in two equivalent ways depending on the employed setup configuration. For the case without the spectral filtering of the etalon, we acquire a complete list of timestamps from all channels and use them to construct the threefold coincidences histograms within a correlation time of $100 \mathrm{~ns}$. For the measurements performed with the etalon filter, due to the longer integration time and the large amount of collected timestamps, the detection events are analyzed in real-time and reduced to the threefold coincidences within a maximum time delay of $0.6 \mathrm{~ns}$. The temporal window is not chosen with the purpose of improving the efficiency of the protocol by temporal filtering. In fact, this strategy is not particularly effective here given the fact that the detector time jitter is higher than the emission lifetimes, no matter how narrow the time gating we could not achieve an improvement in HOM visibility comparable with the one obtained with spectral filtering. Moreover, the window is large enough to discard only a small fraction of the coincidence events. For the entanglement measurement, less than $8 \%$ of the potential coincidences have been excluded with a change of only 0.02 in the entanglement fidelity, comparable with the measurement error. The time window was chosen instead to minimize the number of accidental threefold coincidences due to events in which the early and late photon enter the BSM beam splitter from the same side (corresponding to the side peaks in the HOM histogram), while discarding a minimal number of potential teleportation events. Eight combinations of channels are recorded, stemming from the two possible outputs of the quantum state tomography on the XX photons and from the four possible ways of probing the measurement of a Bell state on the $X$ photons, two for the state $\left|\psi^{-}\right\rangle$and the other two for $\left|\psi^{+}\right\rangle$. The polarization density matrices are built using the direct inversion singlequbit tomography method ${ }^{41}$, which always returns a physical state for the data collected in our experiment.

\section{DATA AVAILABILITY}

The data that support the plots within this paper and the other findings of this study are available from the corresponding authors upon reasonable request.

\section{CODE AVAILABILITY}

The code written for the model simulation within this paper is available from the corresponding authors upon reasonable request.

Received: 5 June 2020; Accepted: 20 November 2020; Published online: 22 January 2021

\section{REFERENCES}

1. Boschi, D., Branca, S., De Martini, F., Hardy, L. \& Popescu, S. Experimental realization of teleporting an unknown pure quantum state via dual classical and Einstein-Podolsky-Rosen channels. Phys. Rev. Lett. 80, 1121-1125 (1998).
2. Bouwmeester, D. et al. Experimental quantum teleportation. Nature 390, 575-579 (1997).

3. Pirandola, S., Eisert, J., Weedbrook, C., Furusawa, A. \& Braunstein, S. L. Advances in quantum teleportation. Nat. Photonics 9, 641-652 (2015).

4. Wehner, S., Elkouss, D. \& Hanson, R. Quantum internet: a vision for the road ahead. Science 362, eaam9288 (2018).

5. Cacciapuoti, A. S. et al. Quantum internet: networking challenges in distributed quantum computing. IEEE Netw. 34, 137-143 (2020).

6. Ren, J. G. et al. Ground-to-satellite quantum teleportation. Nature 549, 70-73 (2017).

7. Simon, C. et al. Quantum repeaters with photon pair sources and multimode memories. Phys. Rev. Lett. 98, 190503 (2007).

8. Takeoka, M., Jin, R.-B. \& Sasaki, M. Full analysis of multi-photon pair effects in spontaneous parametric down conversion based photonic quantum information processing. N. J. Phys. 17, 043030 (2015).

9. Jöns, K. D. et al. Bright nanoscale source of deterministic entangled photon pairs violating Bell's inequality. Sci. Rep. 7, 1700 (2017).

10. Nilsson, J. et al. Quantum teleportation using a light-emitting diode. Nat. Photonics 7, 311-315 (2013).

11. Huwer, J. et al. Quantum-dot-based telecommunication-wavelength quantum relay. Phys. Rev. Appl. 8, 024007 (2017).

12. Anderson, M. et al. Quantum teleportation using highly coherent emission from telecom C-band quantum dots. npj Quantum Inf. 6, 14 (2020).

13. Reindl, M. et al. All-photonic quantum teleportation using on-demand solid-state quantum emitters. Sci. Adv. 4, eaau1255 (2018).

14. Basso Basset, F. et al. Entanglement swapping with photons generated on demand by a quantum dot. Phys. Rev. Lett. 123, 160501 (2019).

15. Zopf, M. et al. Entanglement swapping with semiconductor-generated photons violates Bell's inequality. Phys. Rev. Lett. 123, 160502 (2019).

16. Reindl, M. et al. Phonon-assisted two-photon interference from remote quantum emitters. Nano Lett. 17, 4090-4095 (2017).

17. Weber, J. H. et al. Two-photon interference in the telecom C-band after frequency conversion of photons from remote quantum emitters. Nat. Nanotechnol. 14, 23-26 (2019).

18. Scarani, V. et al. The security of practical quantum key distribution. Rev. Mod. Phys. 81, 1301-1350 (2009).

19. Huber, D. et al. Highly indistinguishable and strongly entangled photons from symmetric GaAs quantum dots. Nat. Commun. 8, 15506 (2017).

20. Basso Basset, F. et al. High-yield fabrication of entangled photon emitters for hybrid quantum networking using high-temperature droplet epitaxy. Nano Lett. 18, 505-512 (2018).

21. Huber, D. et al. Strain-tunable GaAs quantum dot: a nearly dephasing-free source of entangled photon pairs on demand. Phys. Rev. Lett. 121, 033902 (2018).

22. Akopian, N., Wang, L., Rastelli, A., Schmidt, O. G. \& Zwiller, V. Hybrid semiconductor-atomic interface: slowing down single photons from a quantum dot. Nat. Photonics 5, 230-233 (2011).

23. Huang, $H$. et al. Electrically-pumped wavelength-tunable gaas quantum dots interfaced with rubidium atoms. ACS Photonics 4, 868-872 (2017).

24. Kumar, S. et al. Strain-induced tuning of the emission wavelength of high quality GaAs/AlGaAs quantum dots in the spectral range of the $87 \mathrm{Rb} D 2$ lines. Appl. Phys. Lett. 99, 161118 (2011).

25. Namazi, M., Kupchak, C., Jordaan, B., Shahrokhshahi, R. \& Figueroa, E. Ultralownoise room-temperature quantum memory for polarization qubits. Phys. Rev. Appl. 8, 034023 (2017)

26. Wolters, J. et al. Simple atomic quantum memory suitable for semiconductor quantum dot single photons. Phys. Rev. Lett. 119, 060502 (2017).

27. Trotta, R., Martín-Sánchez, J., Daruka, I., Ortix, C. \& Rastelli, A. Energy-tunable sources of entangled photons: a viable concept for solid-state-based quantum relays. Phys. Rev. Lett. 114, 150502 (2015).

28. Gurioli, M., Wang, Z., Rastelli, A., Kuroda, T. \& Sanguinetti, S. Droplet epitaxy of semiconductor nanostructures for quantum photonic devices. Nat. Mater. 18, 799-810 (2019).

29. Basso Basset, F. et al. Spectral broadening in self-assembled GaAs quantum dots with narrow size distribution. J. Appl. Phys. 126, 024301 (2019).

30. Rota, M. B., Basso Basset, F., Tedeschi, D. \& Trotta, R. Entanglement teleportation with photons from quantum dots: towards a solid-state based quantum network. IEEE J. Sel. Top. Quantum Electron. 26, 6400416 (2020).

31. Schimpf, C. et al. Resolving the temporal evolution of line broadening in single quantum emitters. Opt. Express 27, 35290 (2019).

32. Abbarchi, M. et al. Spectral diffusion and line broadening in single self-assembled GaAs/AlGaAs quantum dot photoluminescence. Appl. Phys. Lett. 93, 162101 (2008).

33. Simon, C. \& Poizat, J. P. Creating single time-bin-entangled photon pairs. Phys. Rev. Lett. 94, 28-31 (2005).

34. Huber, T. et al. Measurement and modification of biexciton-exciton time correlations. Opt. Express 21, 9890 (2013). 
35. Schöll, E. et al. Crux of using the cascaded emission of a three-level quantum ladder system to generate indistinguishable photons. Phys. Rev. Lett. 125, 233605 (2020).

36. Ziarkash, A. W., Joshi, S. K., Stipčević, M. \& Ursin, R. Comparative study of afterpulsing behavior and models in single photon counting avalanche photo diode detectors. Sci. Rep. 8, 5076 (2018).

37. Lütkenhaus, N., Calsamiglia, J. \& Suominen, K.-A. Bell measurements for teleportation. Phys. Rev. A 59, 3295-3300 (1999).

38. Kim, Y.-H. H., Kulik, S. P. \& Shih, Y. Quantum teleportation of a polarization state with a complete bell state measurement. Phys. Rev. Lett. 86, 1370-1373 (2001).

39. Nölleke, C. et al. Efficient teleportation between remote single-atom quantum memories. Phys. Rev. Lett. 110, 140403 (2013).

40. Tsurumoto, K., Kuroiwa, R., Kano, H., Sekiguchi, Y. \& Kosaka, H. Quantum teleportation-based state transfer of photon polarization into a carbon spin in diamond. Commun. Phys. 2, 74 (2019).

41. Schmied, R. Quantum state tomography of a single qubit: comparison of methods. J. Mod. Opt. 63, 1744-1758 (2016).

42. Massar, S. \& Popescu, S. Optimal extraction of information from finite quantum ensembles. Phys. Rev. Lett. 74, 1259-1263 (1995).

43. Nielsen, M. A. \& Chuang, I. L. Quantum Computation and Quantum Information Ch 8.4.2. (Cambridge University Press, 2010).

44. Nielsen, M. A. A simple formula for the average gate fidelity of a quantum dynamical operation. Phys. Lett. A 303, 249-252 (2002).

45. Iles-Smith, J., McCutcheon, D. P. S., Nazir, A. \& Mørk, J. Phonon scattering inhibits simultaneous near-unity efficiency and indistinguishability in semiconductor single-photon sources. Nat. Photonics 11, 521-526 (2017).

46. Stevenson, R. M. et al. Quantum teleportation of laser-generated photons with an entangled-light-emitting diode. Nat. Commun. 4, 2859 (2013).

47. Hudson, A. J. et al. Coherence of an entangled exciton-photon state. Phys. Rev. Lett. 99, 266802 (2007).

48. Chau, H. F. Practical scheme to share a secret key through a quantum channel with a $27.6 \%$ bit error rate. Phys. Rev. A 66, 060302 (2002).

49. Shor, P. W. \& Preskill, J. Simple proof of security of the BB84 quantum key distribution protocol. Phys. Rev. Lett. 85, 441-444 (2000).

50. Wang, $\mathrm{H}$. et al. Boson Sampling with 20 Input Photons and a 60-Mode Interferometer in a 1014 -Dimensional Hilbert Space. Phys. Rev. Lett. 123, 250503 (2019).

51. Trotta, R. et al. Wavelength-tunable sources of entangled photons interfaced with atomic vapours. Nat. Commun. 7, 10375 (2016).

52. Huber, D., Reindl, M., Aberl, J., Rastelli, A. \& Trotta, R. Semiconductor quantum dots as an ideal source of polarization-entangled photon pairs on-demand: a review. J. Opt. 20, 073002 (2018).

53. Aspect, A., Grangier, P. \& Roger, G. Experimental tests of realistic local theories via Bell's theorem. Phys. Rev. Lett. 47, 460-463 (1981).

54. Edamatsu, K. Entangled photons: generation, observation, and characterization Jpn J. Appl. Phys. 46, 7175-7187 (2007).

55. Yin, C. et al. Bright-Exciton Fine-Structure Splittings in Single Perovskite Nanocrystals. Phys. Rev. Lett. 119, 026401 (2017).

56. Utzat, $\mathrm{H}$. et al. Coherent single-photon emission from colloidal lead halide perovskite quantum dots. Science 363, 1068-1072 (2019).

57. Nordén, B. Entangled photons from single atoms and molecules. Chem. Phys. 507, 28-33 (2018).

58. Rezai, M., Wrachtrup, J. \& Gerhardt, I. Polarization-entangled photon pairs from a single molecule. Optica 6, 34 (2019).

59. Dotti, N. et al. Germanium-based quantum emitters towards a time-reordering entanglement scheme with degenerate exciton and biexciton states. Phys. Rev. $B$ 91, 205316 (2015).

60. Aharonovich, I., Englund, D. \& Toth, M. Solid-state single-photon emitters. Nat Photonics 10, 631-641 (2016).

61. He, Y.-M. et al. Cascaded emission of single photons from the biexciton in monolayered WSe2. Nat. Commun. 7, 13409 (2016).

62. Tonndorf, P. et al. Single-photon emitters in GaSe. 2D Mater. 4, 021010 (2017)

63. Liu, J. et al. A solid-state source of strongly entangled photon pairs with high brightness and indistinguishability. Nat. Nanotechnol. 14, 586-593 (2019).

64. Wang, $\mathrm{H}$. et al. On-demand semiconductor source of entangled photons which simultaneously has high fidelity, efficiency, and indistinguishability. Phys. Rev. Lett. 122, 113602 (2019).
65. Portalupi, S. L., Jetter, M. \& Michler, P. InAs quantum dots grown on metamorphic buffers as non-classical light sources at telecom C-band: a review. Semicond. Sci. Technol. 34, 053001 (2019).

66. Heyn, C. et al. Highly uniform and strain-free GaAs quantum dots fabricated by filling of self-assembled nanoholes. Appl. Phys. Lett. 94, 183113 (2009).

67. Huo, Y. H., Rastelli, A. \& Schmidt, O. G. Ultra-small excitonic fine structure splitting in highly symmetric quantum dots on GaAs (001) substrate. Appl. Phys. Lett. 102, 152105 (2013).

68. Müller, M., Bounouar, S., Jöns, K. D., Glässl, M. \& Michler, P. On-demand generation of indistinguishable polarization-entangled photon pairs. Nat. Photonics 8 224-228 (2014).

69. Santori, C., Fattal, D., Vučković, J., Solomon, G. S. \& Yamamoto, Y. Indistinguishable photons from a single-photon device. Nature 419, 594-597 (2002).

70. Legero, T., Wilk, T., Kuhn, A. \& Rempe, G. Time-resolved two-photon quantum interference. Appl. Phys. B 77, 797-802 (2003).

\section{ACKNOWLEDGEMENTS}

This work was financially supported by the European Research Council (ERC) under the European Union's Horizon 2020 Research and Innovation Programmes (SPQRel, grant agreement no. 679183), ASCENT+ (grant agreement no. 871130), the Linz Institute of Technology (LIT) through the LIT Secure and Correct Systems Lab funded by the state of Upper Austria and the Austrian Science Fund (FWF): P29603.

\section{AUTHOR CONTRIBUTIONS}

F.B.B. and F.S. performed measurements and data analysis with help from R.T. L.S. K.D.J. helped with investigating the role of the etalon and with initial measurements. M.B.R., D.T., and E.R. contributed to the preparation of the setup. S.F.C.d.S. and A.R. designed and grew the sample. F.B.B., F.S., and R.T. wrote the manuscript with feedback from all authors. All the authors participated in the discussion of the results. R.T. conceived the experiments and coordinated the project.

\section{COMPETING INTERESTS}

The authors declare no competing interests.

\section{ADDITIONAL INFORMATION}

Supplementary information is available for this paper at https://doi.org/10.1038/ s41534-020-00356-0.

Correspondence and requests for materials should be addressed to F.B.B. or R.T.

Reprints and permission information is available at http://www.nature.com/ reprints

Publisher's note Springer Nature remains neutral with regard to jurisdictional claims in published maps and institutional affiliations.

\section{(i)}

Open Access This article is licensed under a Creative Commons Attribution 4.0 International License, which permits use, sharing, adaptation, distribution and reproduction in any medium or format, as long as you give appropriate credit to the original author(s) and the source, provide a link to the Creative Commons license, and indicate if changes were made. The images or other third party material in this article are included in the article's Creative Commons license, unless indicated otherwise in a credit line to the material. If material is not included in the article's Creative Commons license and your intended use is not permitted by statutory regulation or exceeds the permitted use, you will need to obtain permission directly from the copyright holder. To view a copy of this license, visit http://creativecommons. org/licenses/by/4.0/.

(c) The Author(s) 2022, corrected publication 2022 